\title{
Impact of Standard Deviation and Reflectance of the Measured Surface on Laser Diode- Position Sensitive Detector System
}

\author{
Dianyou Song, Dan Xia ${ }^{\mathrm{a}}$ and Zhiyong Wang \\ School of Electronic Information Engineering, Tianjin Key Laboratory of Film Electronic and Communication Device, Tianjin \\ University of Technology, Tianjin 300384, China
}

\begin{abstract}
For improving the accuracy and applicability of laser diode- position sensitive detector (LD-PSD) system, a model of roughness and reflectance of the measured surface is established. Combining this model with the light intensity response model of PSD and the space radiation model, we derived the relationship between the standard deviation $(\sigma)$, reflectance $(\rho)$ and the center-of-gravity of light intensity $\left(X_{0}\right)$. This relationship shows that $X_{0}$ is disturbed by $\sigma$ or $\rho$ only if $\sigma$ or $\rho$ changes within the spot. We conducted a simulation and experiment to test this relationship. The results showed a good consistency with the theory, which proved that the variation of $\sigma$ and $\rho$ can greatly impact the accuracy and robustness of LD-PSD system, and a smaller light spot can improve this situation.
\end{abstract}

\section{Introduction}

Due to its wider spectral range, higher detection precision, higher response rate and simpler processing circuit, position sensitive detector (PSD) has received extensive attention in recent years and is widely used in a variety of measurement systems [1-5]. Typically the PSD is combined with the laser diode (LD) to detect micro-displacement, which is the so-called LD-PSD triangulation system. Previous researchers have conducted many studies on the properties of LD-PSD system. Rainer G.Dorsch et al. studied the uncertainty introduced by laser speckle in laser triangulation measurement [6]. C.Narayannan et al. analyzed the response of PSD under the modulated laser pulse [7]. Song Cui et al. proposed an algorithm to improve the linearity of the PSD response to the incident spot location [8]. And they analyzed the impact of the Gaussian spot size on the measurement [9]. But there is no deep research on the impact of the standard deviation and reflectance of the measured surface. In the research of high precision LD-PSD system, we found that large errors existed in measuring planes with different surface properties. So we estimated an optical model to derive the relationship between these properties of measured surface and the response of PSD, which was proved in our simulation and experiments.

The structure of this paper is as follows. The second section will introduce the measurement principle of the LD-PSD system. In the third section we will propose the optical model of system. And the relationship between the standard deviation, reflectance and the focus of light intensity will be derived by this model. Simulations and experiments are carried out in section 4 and section 5 respectively. Finally, we will summarize our study in section 6.

\section{LD-PSD Systems}

The schematic of LD-PSD system is shown in Figure 1. Monochromatic light issued by LD is transported through the focusing lens, and converges to the measured surface, which is reflected by the plane $P_{1}$. Diffuse reflection light collected by the imaging lens converges to the photosensitive surface of PSD to form a spot $\mathrm{Q}_{1}$. The PSD determines the location of $\mathrm{P}_{1}$ by detecting the position of $\mathrm{Q}_{1}$. When the measured surface has a vertical displacement $\Delta h, \mathrm{Q}_{1}$ will move to the position of $\mathrm{Q}_{2}$. And the PSD determines the location of $\mathrm{P}_{2}$ by detecting the position of $\mathrm{Q}_{2}$. By comparing the photocurrent of the two spots, the system can calculate the displacement $\Delta h$. In order to ensure a constant object-image relationship with the movement of the measured plane, the position of the optical devices is required to meet the Scheimpflug principle. That is, in the observation plane as shown in Figure 1, the optical axis of LD, the principle plane of the imaging lens and the direction of the photosensitive surface of PSD should intersect at point $\mathrm{O}$.

The key of LD-PSD system is the detection of spot position with PSD. So we need to introduce the principle of PSD. PSD is a kind of detectors based on the lateral photoeffect. When the light projects on the photosensitive surface of PSD, the two electrodes generate photocurrent $i_{\text {left }}$ and $i_{\text {right }}$ separately. Then we can derive the position of spot from the following equation:

$$
X_{0}=\frac{L}{2}\left(1-\frac{i_{\text {left }}-i_{\text {right }}}{i_{\text {left }}+i_{\text {right }}}\right)
$$

\footnotetext{
${ }^{a}$ Corresponding author: xiadan@tjut.edu.cn
} 
where $L$ is the length of the photosensitive surface of PSD. Typically, the spot size on the PSD cannot be ignored compared to the photosensitive surface size of PSD. In this paper, we use the model proposed by Lingchen Kong et al to divide the real spot into a series of small spots [10]. The size of each small spot can be ignored compared to $L$. So the generated photocurrent of these points is a linear superposition of each photocurrent generated by small points. In this model, the photocurrent of the two electrodes is as follows:

$$
\begin{aligned}
& i_{\text {left }}=D \int_{X_{l}}^{X_{r}} \Phi(X)\left(1-\frac{X}{L}\right) d X \\
& i_{\text {right }}=D \int_{X_{l}}^{X_{r}} \Phi(X) \frac{X}{L} d X
\end{aligned}
$$

where $D$ is the generation rate of the electron-hole pairs induced by per unit light intensity, $\Phi(X)$ is the incident radiant flux at the point $X, X_{l}$ and $X_{r}$ are the left and right margins of the spot position. Take $i_{\text {left }}$ and $i_{\text {right }}$ into (1), $X_{0}$ can be written as:

$$
X_{0}=\frac{\int_{X_{l}}^{X_{r}} \Phi(X) X d X}{\int_{X_{l}}^{X_{r}} \Phi(X) d X}
$$

The equation (4) shows that the PSD detection position is center-of-gravity of light intensity.

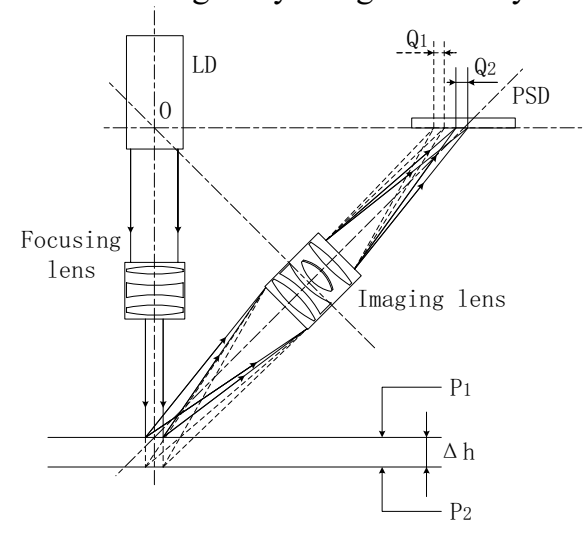

Figure 1. Schematic of LD-PSD system.

\section{Theoretical analyses}

There are three radiation models when light is irradiated to the interface. They are:

(1) When the interface is a smooth surface, the light produces only two types of behavior-absorption and specular reflection, as shown in Figure 2(a). Here, smooth surface is a plane whose standard deviation of height is far less than the wavelength of incident light.

(2) When the interface is a rough surface, the light also produces two types of behavior-absorption and diffuse reflection [11], as shown in Figure 2(b). This radiation model is commonly used in rough dielectric surfaces such as walls and ceramics.
(3) In most cases, the light at the interface produces four types of behavior-absorption, specular reflection, specular diffuse lobe and diffuse reflection [12], as shown in Figure 2(c). Generally, the object surface of LD-PSD system belongs to this model.

For the system as shown in Fig.1, Monochromatic light is vertically focused onto the measured plane. So the angles of incident and reflection are $0^{\circ}$. Contrasting Figure 1 with Figure 2(c), we can obtain that specular reflection light and specular diffuse lobe is close to the normal. So the imaging lens whose angle of axis and the normal is $45^{\circ}$ can only receive the diffuse reflection light. Based on the micro-surface model proposed by Oren and Nayar [13], the region receiving light of the measured surface can be divided into many micro-surfaces. The diffuse reflection light of each micro-surface follows Lambert Law:

$$
L_{r}=\frac{1}{\pi} L_{i} \rho \cos \theta_{i} d \omega
$$

where $L$ is the radiance of receiving direction, $\theta$ is the direction angle of receiving, $\omega$ is the solid angle of receiver and $\rho$ is the reflectance of micro-surface.

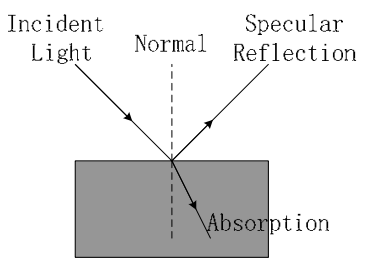

(a) The behaviour of light when it irradiates to an ideal smooth interface.

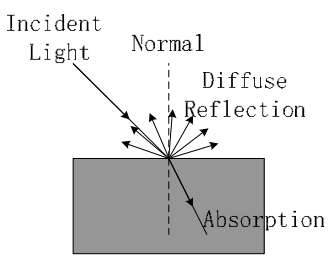

(b) The behaviour of light when it irradiates to an ideal rough interface.

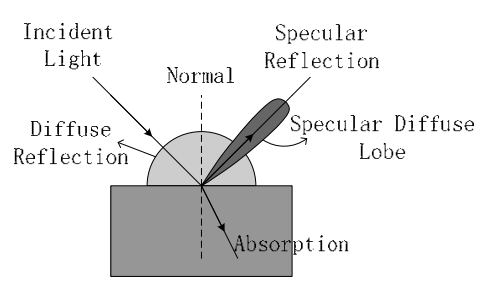

(c) The behaviour of light when it irradiates to the real interface.

Figure 2. Absorption-reflection model of interface.

Lawrence B. Wolff et al. have studied the diffuse reflected radiance of Gaussian distribution microfacet orientations, which is superposition of masking, shadowing and inter-reflection as follows[14]:

$$
\begin{aligned}
L_{r}\left(\theta_{r}, \theta_{i}, \phi_{r}-\phi_{i} ; \sigma\right) & =L_{r}^{1}\left(\theta_{r}, \theta_{i}, \phi_{r}-\phi_{i} ; \sigma\right) \\
& +L_{r}^{2}\left(\theta_{r}, \theta_{i}, \phi_{r}-\phi_{i} ; \sigma\right)
\end{aligned}
$$




$$
\begin{gathered}
L_{r}^{1}\left(\theta_{r}, \theta_{i}, \phi_{r}-\phi_{i} ; \sigma\right)=\frac{\rho}{\pi} L_{i} \cos \theta_{i}\left[C_{1}(\sigma)\right. \\
+\cos \left(\phi_{r}-\phi_{i}\right) C_{2}\left(\alpha ; \beta ; \phi_{r}-\phi_{i} ; \sigma\right) \tan \beta \\
\left.+\left(1-\left|\cos \left(\phi_{r}-\phi_{i}\right)\right|\right) C_{3}(\alpha ; \beta ; \sigma) \tan \left(\frac{\alpha+\beta}{2}\right)\right] \\
L_{r}^{2}\left(\theta_{r}, \theta_{i}, \phi_{r}-\phi_{i} ; \sigma\right)=0.17 \frac{\rho^{2}}{\pi} L_{i} \cos \theta_{i} \frac{\sigma^{2}}{\sigma^{2}+0.13} \\
\quad \times\left[1-\cos \left(\phi_{r}-\phi_{i}\right)\left(\frac{2 \beta}{\pi}\right)^{2}\right] \\
C_{1}=1-0.5 \frac{\sigma^{2}}{\sigma^{2}+0.33} \\
0.45 \frac{\sigma^{2}}{\sigma^{2}+0.09} \sin \alpha \\
0.45 \frac{\sigma^{2}}{\sigma^{2}+0.09}\left(\sin \alpha-\left(\frac{2 \beta}{\pi}\right)^{3}\right) \quad \text { otherwise } \\
C_{3}=0.125\left(\frac{\sigma^{2}}{\sigma^{2}+0.09}\right)\left(\frac{4 \alpha \beta}{\pi^{2}}\right)^{2} \\
\alpha=\max \left[\theta_{r}, \theta_{i}\right] \\
\beta=\min \left[\theta_{r}, \theta_{i}\right]
\end{gathered}
$$

where the definitions of $\theta_{i}, \theta_{r}, \varphi_{i}$ and $\varphi_{r}$ are shown in Figure $3, \sigma$ is the standard deviation of Gaussian distribution micro-surfaces, which is used to characterize the surface roughness [15-19]. In Ld-PSD system shown in Figure1, it can be derived that:

$$
\theta_{i}=0, \theta_{r}=\pi / 4, \varphi_{i}=\varphi_{r}=\pi / 2
$$

Take these angles into (6) (13), the radiance of diffuse reflection can be written as follows:

$$
L_{r}=\frac{\rho}{\pi} L_{i}\left(1-0.5 \frac{\sigma^{2}}{\sigma^{2}+0.33}\right)+0.17 \frac{\rho^{2}}{\pi} L_{i} \frac{\sigma^{2}}{\sigma^{2}+0.13}
$$

The relationship between radiance and radiant flux is:

$$
L_{r}=\frac{d^{2} \Phi_{e}}{d \Omega d A \cos \theta}
$$

where $\Phi_{e}$ is radiant flux, $d A$ is the area of receiving surface, $d \Omega$ is solid angle at the given direction to light source and $\theta$ is the angle between the given direction and the normal of receiving surface. In LD-PSD system shown in Figure 1, the imaging lens collect diffuse reflection light and project to PSD. Therefore, radiant flux received by PSD is equal to radiant flux collected by imaging lens. Here, we assume that the receiving area of imaging lens is $A$. Divide $A$ into the combination of micro-area $A_{n}$. The direction angle and the solid angle corresponding to $A_{n}$ are $\theta_{n}$ and $\Omega_{n}$. Then radiant flux received by PSD can be derived from equation (15) as follows:

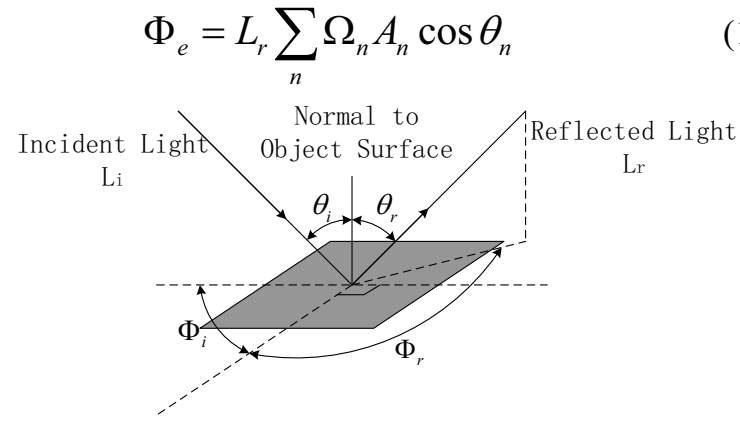

Figure 3. Definition of diffuse reflection parameters

The above analysis is based on the condition that the light spot on measured surface is an ideal point. However, as described in section 2, the light spot of LD has a size that cannot be ignored. For this reason, we divide the light spot into a series of micro-spots. The diffuse reflection radiant flux of each micro-spot obeys equation (16). So we can take $\Phi_{e}$ as a function of $X$ into equation (4), where $X$ is the position of micro-spot at the photosensitive surface of PSD.

$$
\begin{aligned}
X_{0} & =\left\{\int _ { X _ { l } } ^ { X _ { r } } \left[\rho(X)\left(1-0.5 \frac{\sigma(X)^{2}}{\sigma(X)^{2}+0.33}\right)\right.\right. \\
& \left.\left.+0.17 \rho(X)^{2} \frac{\sigma(X)^{2}}{\sigma(X)^{2}+0.13}\right] X d X\right\} \\
& /\left\{\int _ { X _ { l } } ^ { X _ { r } } \left[\rho(X)\left(1-0.5 \frac{\sigma(X)^{2}}{\sigma(X)^{2}+0.33}\right)\right.\right. \\
& \left.\left.+0.17 \rho(X)^{2} \frac{\sigma(X)^{2}}{\sigma(X)^{2}+0.13}\right] d X\right\}
\end{aligned}
$$

where $\rho(X)$ and $\sigma(X)$ are reflectance and standard deviation of the measured surface corresponding to $X$. Equation (17) implies that center-of-gravity of light spot detected by LD-PSD system is related to the reflectance and standard deviation of measured surface. When LDPSD system scans the measured surface, the light spot will move, while $\rho$ and $\sigma$ are variables. This will introduce uncertainty of $X_{0}$ to the measured result, which decreases the stability and robustness of LD-PSD system. However, if we can ensure that $\rho$ and $\sigma$ are constants between $X_{l}$ and $X_{r}$ at each moment while scanning, $X_{0}$ will no longer be related to $\rho$ and $\sigma$. Then the system will get high stability and robustness.

\section{Simulations}

The reflectance and standard deviation of measured surface always change simultaneously. So we assume that $t\left(X_{l}<t<X_{r}\right)$ is the critical point of the changing of $\rho$ and $\sigma$. That is, in equation (17), $\rho(X)$ and $\sigma(X)$ are $\rho_{1}$ and $\sigma_{1}$ when $X_{l}<X<t$, while $\rho(X)$ and $\sigma(X)$ are $\rho_{2}$ and $\sigma_{2}$ when $t<X<X_{r}$. Under this assumption, equation (17) can be written as:

$$
X_{0}=\frac{(a-b) t^{2}-a X_{l}^{2}+b X_{r}^{2}}{2(a-b) t-2 a X_{l}+2 b X_{r}}
$$


where

$$
\begin{aligned}
& a=\rho_{1}\left(1-0.5 \frac{\sigma_{1}}{\sigma_{1}+0.33}\right)+0.17 \rho_{1} \frac{\sigma_{1}^{2}}{\sigma_{1}^{2}+0.13} \\
& b=\rho_{2}\left(1-0.5 \frac{\sigma_{2}}{\sigma_{2}+0.33}\right)+0.17 \rho_{2} \frac{\sigma_{2}^{2}}{\sigma_{2}^{2}+0.13}
\end{aligned}
$$

Then we can derive the drift error of center-ofgravity of light spot as:

$$
\Delta X=X_{0}-\frac{X_{l}+X_{r}}{2}=\frac{(a-b)\left(t-X_{l}\right)\left(t-X_{r}\right)}{2(a-b) t-2 a X_{l}+2 b X_{r}}
$$

From equation (21) we can derive that $a=b$ and $\Delta X$ $=0$ when $\rho_{1}=\rho_{2}$ and $\sigma_{1}=\sigma_{2}$, which is corresponding to the condition that $\rho$ and $\sigma$ are constants within the light spot. And when $\rho_{1} \neq \rho_{2}$ or $\sigma_{1} \neq \sigma_{2}, a$ is no longer equal to $b$ and $\Delta X$ changes with the change of $t$. The relationship between $t$ and $\Delta X$ is depicted in Figure 4, where the horizontal axis is the position of critical point $t$ while the vertical axis is the drift $\Delta X$. The blue curve indicates that the light spot diameter is $D$, while the red curve indicates that the light spot diameter is $2 D$. For the blue curve, when $t$ is located out of the spot $(1<t / D<2), \Delta X$ is 0 . While $\Delta X$ is no longer 0 when $t$ is located within the light spot $(0<t / D<1)$ and the variation trend is similar to the quadratic curve. Contrasted with the blue curve, the red curve has a larger range of error because of the larger spot diameter.

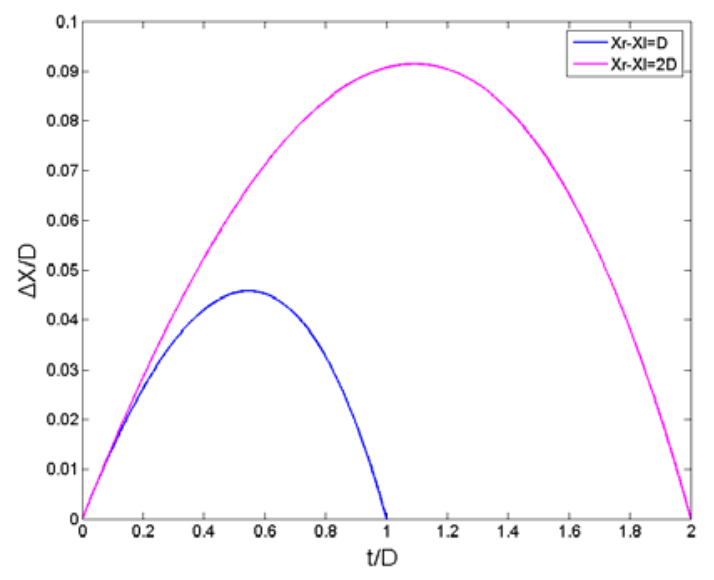

Figure 4. Simulation result of critical point position and errors.

\section{Experiments}

Before the experiment, we need to exclude the influence of other factors on the measurement results. Take (16) into (2) and (3):

$$
\begin{aligned}
i_{\text {left }} & =D \int_{X_{l}}^{X_{r}}\left[\frac{\rho(X)}{\pi} L_{i}\left(1-0.5 \frac{\sigma(X)^{2}}{\sigma(X)^{2}+0.33}\right)\right. \\
& \left.+0.17 \frac{\rho(X)^{2}}{\pi} L_{i} \frac{\sigma(X)^{2}}{\sigma(X)^{2}+0.13} \sum_{n} \Omega_{n} A_{n} \cos \theta_{n}\right]\left(1-\frac{X}{L}\right) d X
\end{aligned}
$$

$$
\begin{aligned}
i_{\text {rignt }} & =D \int_{X_{l}}^{X_{r}}\left[\frac{\rho(X)}{\pi} L_{i}\left(1-0.5 \frac{\sigma(X)^{2}}{\sigma(X)^{2}+0.33}\right)\right. \\
& \left.+0.17 \frac{\rho(X)^{2}}{\pi} L_{i} \frac{\sigma(X)^{2}}{\sigma(X)^{2}+0.13} \sum_{n} \Omega_{n} A_{n} \cos \theta_{n}\right] \frac{X}{L} d X
\end{aligned}
$$

The above two equations shows that photocurrent of PSD is also related to reflectance and standard deviation of measured surface. The A/D converter of LD-PSD signal processing circuit has a linear conversion range. When photocurrent changes the signal may exceed the linear conversion range, which will lead to a big circuit noise and the measured result is not the real position of $X_{0}$. So we use the signal band-pass filtering techniques to optimize the circuit system, which can modulate the intensity of photocurrent adaptively. The frequency response of band-pass filter is as follows:

$$
A_{u}=\left|\frac{1}{1+j Q\left(\frac{f}{f_{0}}-\frac{f_{0}}{f}\right)}\right|
$$

where $f_{0}$ is the natural frequency and $f$ is the modulate frequency. When the circuit finds the change of photocurrent, $f$ will change adaptively through negative feedback to modulate the photocurrent. Finally, photocurrent will rapidly return to the linear region of the A/D converter. This allows the circuit system and the optical system to maintain a good consistency, thus excluding the impact of the circuit factors.

In the experiment, we use a standard roughness gauge block as the measured object. The block length is $L$ and the flatness error is less than $1 \mu \mathrm{m}$. The centre line divides the roughness of block into $\mu 1$ and $\mu 2$. So the reflectance and standard deviation are $\rho_{1}, \sigma_{1}$ and $\rho_{2}, \sigma_{2}$ separately. We set the real distance of block to $50 \mathrm{~mm}(d$ $=50 \mathrm{~mm}$ ) and use LD-PSD system to scan the block from left boundary to right. The resolution of LD-PSD system is $1 \mu \mathrm{m}$ and the diameter of light spot is $D$ and $2 D(D<<$ $L)$. If the distance from spot right margin to the left boundary of block is $x$, the distance of the block measured by LD-PSD is depicted in Figure 5. For the first scanning, the diameter of light spot is $D$. When $x$ is less than $D$, the measured results have large errors because the light spot has not entirely projected to the block surface. When $x=D$, the measurement starts to be normal and the measured results are equal to the real value. With the moving of the spot, when $x=L / 2$, that is, $\rho$ and $\sigma$ begin to change within the spot, the measured results begin to be error. When $x=L / 2+D$, the measured results return to the real value because the light spot have entirely accessed to the surface that the reflectance is $\rho_{2}$ and standard deviation is $\sigma_{2}$. For the second scanning with the $2 D$ diameter of light spot, the trend of the curve is similar to that of the first scanning. From Figure 5, it can be seen that the trend of curves is agree with the simulation in Figure 4 when the spot goes through the mutative $\sigma$ and $\rho$. And a bigger light spot will have a larger probability of error because the bigger spot is apt to reach the area that contains mutative reflectance and 
standard deviation, meanwhile it is easier to exceed the measured surface.

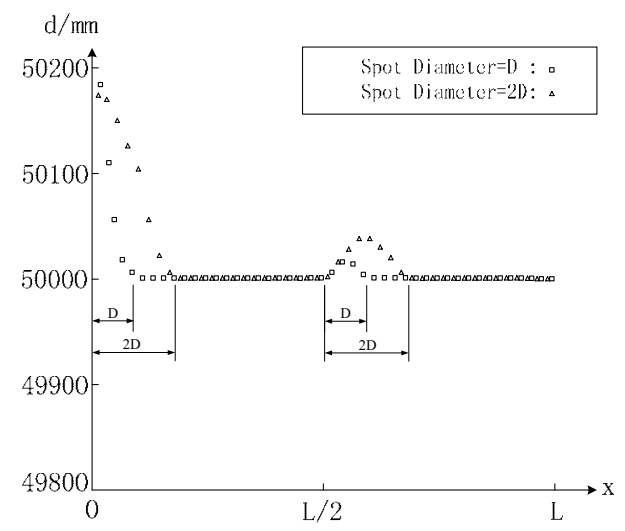

Figure 5. The distance measured by the scanning LD-PSD.

\section{Conclusions}

Starting from the reflection model of the general interface and combining the Lambert law and the metering center-of-gravity of light spot principle of PSD, we derive the relationship between the spot location and the reflectance and standard deviation of measured surface, which allows us to analyze the impact of the measured surface properties on LD-PSD system. By simulations and experiments, we demonstrate that error occurs when reflectance and standard deviation changing within the spot area, while the measured result is stability when reflectance and standard deviation are constant at every moment. In addition, a smaller laser spot can get smaller error and smaller error area. In other words, a smaller laser spot can improve the accuracy and robustness of LD-PSD system.

\section{Acknowledgements}

The research was supported in part by the Yumiao Foundation of Tianjin University of Technology (LGYM201106).

\section{References}

1. J. Contreras, L. Gomes, S. Filonovich, N. Correia, E. Fortunato, R. Martins, I. Ferreira, Opt. Express, 20, 4583-4602 (2012)

2. I. Matsuya, R. Tomishi, M. Sato, K. Kanekawa, Y. Nitta, M. Takahashi, S. Miura, Y. Suzuki, T. Hatada, R. Katamura, T. Tanii, S. Shoji, A. Nishitani, I. Ohdomari, IEEJ T. Electr. Electr., 6, 266-272 (2011)

3. V. Esmaeile Sani, A. Moussavi-Zarandi, M. Kafaee, Nucl. Instrum. Methods Phys. Res., Sect. A, 654, 464-470 (2011)

4. Y.C. Sung, T. Miyasaka, T.K. Lin, C.Y. Wang, C.Y. Wang, Struct. Control Health Monit., 19, 295-308 (2012)

5. H. Nakajima, K. Sumi, H. Inujima, IEEE Trans. Instrum. Meas., 59, 3041-3048 (2010)
6. R.G. Dorsch, G. Hausle, J.M. Herrmann, Appl. Opt., 33, 1306-1314 (1994)

7. C. Narayanan, A.B. Buckman, I. Busch-Vishniac, W. Wang, IEEE T. Electron Dev., 40, 1688-1694 (1993)

8. S. Cui, Y.C. Soh, IEEE T. Electron Dev., 57, 23102316 (2010)

9. S. Cui, Y.C. Soh, Opt. Quant. Electron., 42, 721-728 (2011)

10. L. Kong, E. Li, D. Yang, H. Xing, IEEE T. Electron Dev., 59, 835-840 (2012)

11. K.E. Torrance, E.M. Sparrow, J. Opt. Soc. Am., 57, 1105-1114 (1967)

12. T. Kiuchi, K Ikeuchi, IAPR MAV' 92, 175-178 (1992)

13. M. Oren, S.K. Nayar, Int. J. Comput. Vision, 14, 227-251 (1995)

14. L.B.Wolff, S.K. Nayar, M. Oren, Int. J. Comput. Vision, 30, 55-71 (1998)

15. R. Reizer, Wear, 271, 539-543 (2011)

16. G. Zhang, Z.S. Wu, Opt. Express, 19, 7007-7019 (2011)

17. G. Zhang, Z.S. Wu, Opt. Express, 20, 1491-1502 (2012)

18. J.F. Lingevitch, K.D. Lepage, IEEE J. Oceanic Eng., 35, 199-208 (2010)

19. C.F. Cheng, D.P. Qi, D.L. Liu, S.Y. Teng, Acta Phys. Sinica, 48, 1635-1643 (1999) 